\title{
Patients with cancer often felt a need to conceal their distress to protect family, friends, and doctors
}

\author{
Byrne A, Ellershaw J, Holcombe C, et al. Patients' experience of cancer: evidence of the role of 'fighting' in collusive clinical \\ communication. Patient Educ Couns 2002;48:15-21.
}

\section{QUESTION: How do patients respond to having cancer?}

\section{Design}

Qualitative study.

\section{Setting}

Hospital clinics and a day care at an associated hospice in the UK.

\section{Participants}

30 patients who were $37-88$ years of age (median age 55 y, $60 \%$ women, $100 \%$ white). Patients had cancer of the breast ( $\mathrm{n}=11)$, lung $(\mathrm{n}=4)$, bowel/anus $(\mathrm{n}=6)$, larynx/ tonsil/neck $(n=3)$, cervix $(n=2)$, kidney/liver $(n=2)$, and prostate $(\mathrm{n}=1) .1$ patient had non-Hodgkin's lymphoma. The time since treatment was $\leq 7$ days for 4 patients, $<1$ year for 14 patients, and 1-19 years for 12 patients. 14 patients received curative treatment and 16 palliative treatment.

\section{Methods}

Patients were individually interviewed for 30-180 minutes (mean $65 \mathrm{~min}$ ) about their experience of cancer. Open questions, prompts, and reflection were used to help patients describe personal changes and their experiences of others. Interviews were audiotaped and transcribed. An inductive thematic analysis was done.

\section{Main findings}

(1) Concealing distress. Almost all patients expressed the importance of concealing distress or unhappiness from others. Most often, the desire to conceal distress stemmed from a need to protect family and friends. Sometimes doctors collaborated in the concealment by encouraging patients and family to protect each other. Patients often concealed their distress from doctors to avoid disapproval or embarrassment. (2) Rejecting cancer. Patients commonly used the language of effort and struggle to describe their experiences of cancer. However, their resistance was often directed against the emotional response to cancer, not the disease itself. Although they tended to desire emotional expression, they were constrained in doing so by their own ambivalence, patterns of communication in healthcare relationships, and constraints in the care environment. They also understood that expression was discouraged by the doctors' emphasis on always being positive. Being "normal" was important to many patients. They maintained familiar routines and sought to restore the body to original functioning. Some patients minimised disease by comparing cancer to other challenges or by observing that many people were worse off than they were. (3) Accepting cancer. Patients expressed feeling shock, bewilderment, and searching for a cause. The word "cancer" elicited strong responses, and patients reported feeling dazed for a few days after being told they had cancer. Patients spent time trying to find a reason why the cancer occurred. Fear was a common response. Patients dreaded their next appointment and were afraid of the unknown. Patients who had been treated successfully were afraid of recurrence. For some, this fear was a phase, whereas for others it was a constant struggle. Some patients described the inevitability of the disease or its treatment trajectory. They understood that having cancer was something that one simply had to accept. Some patients responded by assimilating the illness and re-evaluating life. These patients felt that they valued life more because of cancer and believed that their priorities had changed for the better.

\section{Conclusions}

Patients with cancer responded in various ways. Most felt a need to conceal their distress to protect family, friends, and doctors. The desire to fight the disease and clinicians' emphasis on being positive often resulted in patients suppressing their more negative emotional responses to having the disease.
Source of funding:
no external funding.

For correspondence: Dr P Salmon,

Department of Clinical Psychology, University of Liverpool, Liverpool, UK.psalmon@liv.ac.uk

\section{COMMENTARY}

The study by Byrne et al is situated in important debates about clinician-patient communication in cancer care. From a psychological perspective, the authors have focused on individual patient responses to the cancer disease experience, drawing attention to the implications of these responses for clinician-patient communication. The findings of the study highlight the importance of sensitive communication in clinician-patient encounters for enhancing patient wellbeing.

Because of its implications for healthcare communication, the authors' conclusion that patients' positive expressions may be used to conceal their distress and protect others deserves critical attention. Specifically, this finding may lead clinicians to question the authenticity of patients' positive expressions of coping and courage and, perhaps, to interpret them in a simplistic way. For the same reasons, the authors' findings regarding responses that signal rejection of cancer or its symptoms or the acceptance of cancer deserve some scrutiny.

The complex and shifting dynamics that shape human expression were not incorporated into the analysis. As such, temporal, contextual, and relational features of the participants' expressions of responses to cancers are obscured. Previous research has shown the importance of attending to the multiple and complex meanings of illness experiences in healthcare encounters. ${ }^{1}$ Other research is beginning to show that patient responses to cancer may be multifaceted and are as much related to their life circumstances as to the disease itself. ${ }^{2-5}$ Although Byrne et al report some variations among participants, participant comments are largely decontextualised.

Although the study findings are useful in alerting us to alternate ways of thinking about patient responses to disease, the authors' conclusions must be interpreted cautiously in regard to their implications for healthcare practice and considered in the context of the growing body of research addressing communication in cancer care.

Gladys McPherson, RN, MSN Graduate Research Assistant School of Nursing, University of British Columbia Vancouver, British Columbia, Canada

1 Kleinman A. Local worlds of suffering: an interpersonal focus of ethnographies of illness experience. Oual Health Res 1992;2:127-34.

experience. Qual Health Res 1992;2:127-34. Kaplowitz SA, Campo S, Chiu WT. Cancer patients

prognosis information. Health Commun 2002;14:221-41. Mcillmurray MB, Thomas C, Francis B, et al. The psychosocial needs of cancer

findings from an observational study. Eur J Cancer Care (Engl) 2001·10:261-9. Salander P. Bad news from the patient's perspective: an analysis of the written narratives of newly diagnosed cancer patients. Soc Sci Med 2002;55:721-32.

Thorne SE. Communication in cancer care: what science can and cannot teach us. Cancer Nurs 1999;22:370-8. 